\title{
Surgical Management of Localized Hepatocellular Carcinoma in Times of Crisis: A Strategic Approach to Resource Utilization
}

\author{
This article was published in the following Dove Press journal:
}

Journal of Hepatocellular Carcinoma

\author{
Hop Tran Cao \\ Ching-Wei Tzeng \\ Yun Shin Chun \\ Thomas Aloia \\ Jean Nicolas Vauthey
}

Department of Surgical Oncology, The University of Texas MD Anderson Cancer

Center, Houston, TX, USA
Correspondence: Hop Tran Cao Department of Surgical Oncology, The University of Texas MD Anderson Cancer Center, 1400 Pressler St, Unit I484, Houston, TX 77030, USA

Tel +I 7I3745-4670

Fax +1 713745-1921

Email hstran@mdanderson.org

\begin{abstract}
The management of localized hepatocellular carcinoma (HCC) is complex and requires multidisciplinary consideration. In times of crisis when resources are limited and the health of patients might be compromised, as is the case with COVID-19, a strategic approach that takes into account tumor characteristics, patient factors, and available treatment options can optimize patient outcomes while balancing resource utilization. Herein, we detail our group's management strategy for patients with localized HCC during the global pandemic that carefully considers individual patient needs and those of the institutional workforce, the local healthcare system, and the greater patient community we serve.
\end{abstract}

Keywords: hepatocellular carcinoma, COVID-19, resources, multidisciplinary, hepatectomy

Over the past months, the COVID-19 pandemic has laid bare the vulnerabilities of our healthcare system and put to the test its ability to withstand large-scale health crises. To ensure that the system bends but does not break, a two-pronged approach has been deployed. On the public health policy side, measures to slow down the spread of the virus through social distancing and stay-at-home orders in some parts of the country have been implemented to "flatten the curve". On the healthcare delivery side, hospitals have limited on-site foot traffic and postponed elective cases in an effort to remain nimble in their response to anticipated surges of uncertain magnitude and timing. In the midst of these adjustments, oncologists have been compelled to be thoughtful and strategic in their treatment approach to cancer patients, who are especially susceptible to the ravages of the COVID-19. In the case of hepatocellular carcinoma ( $\mathrm{HCC}$ ), this task is made especially challenging because management decisions must take into account not just the characteristics of the tumor, but those of the patient physiology and of the underlying liver condition as well.

As part of our institution's concerted and proactive response to the pandemic, ${ }^{1,2}$ our team formulated surgical strategies for HPB malignancies that were guided by the following principles:

- Cases should be postponed if oncological outcomes were unlikely to be adversely affected by a delay of 8-12 weeks or if an alternative treatment option could safely postpone surgery. The choice of the 8-12 week window delay was based on modeling of the local surge and continually updated to reflect modeling calculations that account for the conditions on the ground. 
- Cases with a high risk for intensive care unit (ICU) utilization, blood transfusion requirement exceeding four units, and/or prolonged hospitalization should be carefully reviewed and postponed if appropriate to minimize taxing finite hospital resources that would be critical during a potential wave of COVID-19 admissions.

- Due to the potential for aerosolization of viral particles with pneumoperitoneum, and following national and institutional guidelines, laparoscopic and robotic cases should employ filtration systems, minimize pneumoperitoneum pressures when possible, and balance any perceived benefit of the approach with the risk of greater exposure with prolonged case duration. ${ }^{3}$

At the same time, any modification to our treatment protocol also needed to take into account our group's surgical experience, where rates of ICU admission and blood transfusion even after major hepatectomy are $<1 \%$ and $<3 \%$, respectively. In dealing with $\mathrm{HCC}$, we developed a management strategy that takes advantage of adjunctive modalities such as transarterial chemoembolization (TACE) or radioembolization (TARE). All decisions are reached with multidisciplinary input, and patients deemed best served with transplantation are managed in accordance with United Network of Organ Sharing (UNOS) guidelines. ${ }^{4}$

At baseline, all patients with localized, non-metastatic, HCC seen at our institution are evaluated by surgeons for consideration of resection. Patients with poor performance status, especially in the context of severe uncontrolled comorbid conditions, are not offered surgery and are instead referred for non-operative therapies with multidisciplinary consensus. Their management falls outside the scope of this article. For those who are fit for surgery, the extent of hepatectomy is largely dictated by severity of the underlying liver disease and liver volumetry.

As a general rule, patients with Child's A cirrhosis can be offered a major hepatectomy, whereas those with Child's B cirrhosis should only be considered for more limited hepatectomies. Child's C cirrhotics are never resection candidates due to the exceedingly high risk of perioperative mortality and morbidity and because it is often the liver disease, rather than the cancer, that determines their clinical prognosis. ${ }^{5}$ It should be pointed out that the Child-Pugh Score does not fully account for portal hypertension. Because of this, it is important to carefully review the patient's chart for evidence of portal hypertension. This includes presence of mesenteric varices, ascites, recanalization of the umbilical vein, splenomegaly, enlarged portal vein diameter, and caudate hypertrophy on cross-sectional imaging, and thrombocytopenia on blood work. Normally, the threshold to obtain esophagogastro-duodenoscopy (EGD) to rule out varices would be low. However, because of the risk of viral transmission during COVID-19, we are leaning more heavily on our Radiology colleagues to make this assessment. If endoscopy is still indicated, safety measures include screening and testing patients for COVID-19 and use of personal protective equipment, including N95 masks, with each procedure. Finally, when suspicion for portal hypertension is high, measurement of portal venous pressure gradient can be performed, with values exceeding $10 \mathrm{~mm} \mathrm{Hg}$ confirming the diagnosis. ${ }^{6}$ The need to rule out portal hypertension is especially critical during times of limited resources because its clinical implications are so significant: portal hypertension not only complicates any intended operation and increases the risk of hemorrhage, it also serves as a marker of limited hepatic function reserve. For these reasons, we approach patients with Child's A cirrhosis and portal hypertension the way we do those with Child's B cirrhosis.

Once liver physiology has been deemed adequate to support a hepatectomy, a number of HCC presentations may be considered for resection in our practice. Based on tumor characteristics and biology, these HCC presentations fall into one of four main categories of management during COVID-19 (or any future times of limited resources), which are detailed below. Of note, patients who meet transplant criteria and are interested in pursuing this option are still referred to our transplant partners for evaluation. We emphasize, as always, the need for an open and honest discussion about the potential advantages and drawbacks of each approach with patients in each case.

- Tumors that can safely be observed with delayed resection

Solitary HCCs that are small $(<2 \mathrm{~cm})$ with no vascular invasion - T1a tumors - and with a normal alphafetoprotein (AFP) level may be safely observed, as their risk for disease progression to the point of adversely impacting the patient's oncologic outcome is low. Although they may also fall under the next category, in our efforts to limit patient and healthcare provider 
exposure, these represent lower priority tumors for intervention.

- Tumors that can be definitively treated with a different treatment modality

Solitary HCCs that are small (up to $3 \mathrm{~cm}$ ), especially those situated centrally, may be safely addressed with definitive ablation if their anatomic relationship to major vessels or bile ducts is favorable. ${ }^{7}$ Tumors with a high AFP take precedence over those with normal AFP values, which may be observed. Careful evaluation and intervention by our dedicated HPB Interventional Radiology team has resulted in outstanding outcomes for these types of tumors.

- Tumors that should be bridged with adjunctive modalities until surgery can be performed

HCCs that are too large to effectively be treated with ablation $(>3 \mathrm{~cm})$ and those that cannot be safely observed due to the presence of high-risk features such as elevated AFP, major vascular invasion on imaging, or multifocality, may be managed with a staged approach that is made especially relevant during times of scarce resources. While some view large tumor size $(>5 \mathrm{~cm})$, multifocal disease, and major vascular invasion as contraindications to surgery, our group instead favors treating these patients with transarterial liver-directed therapy, with TACE or TARE, as a first step before contemplating surgical resection. This can control the disease locally and allows for an evaluation of the tumor biology. The potential role of immunotherapy or targeted therapy in the neoadjuvant setting is a topic of ongoing investigation.

\section{- Tumors that should be resected}

Unlike other cancers for which there are effective neoadjuvant treatment options, no such therapy currently exists for HCC. For this reason, absent a sound bridging strategy or available bridging modality, surgery remains the best option even during this COVID-19 crisis. Patients who are not good candidates for liver-directed therapy due to anatomic constraints (eg, poorly vascularized tumors or those whose blood supply cannot be well isolated), intolerant of liver-directed therapy, or otherwise refuse it are offered surgery.

Once the decision is reached to consider surgery, liver volumetry is routinely obtained as previously described for all patients in whom major hepatectomy would be required. A standardized future liver remnant (sFLR) of $40 \%$ is required for safe resection in the setting of cirrhosis to minimize the risk of post-hepatectomy liver failure. ${ }^{8}$ When inadequate, the sFLR can be induced to hypertrophy with the use of portal vein embolization (PVE). ${ }^{9}$ In addition to its role in liver hypertrophy, PVE also offers surgeons a chance at better patient and liver selection for major hepatectomy by revealing the regenerative potential of the liver and exposing tumor biology. In the event of inadequate liver growth, although attempts at further augmenting the FLR may be considered with the addition of hepatic vein embolization and/ or Yttrium-90 radioembolization, these patients may be better served with non-operative treatment. ${ }^{10,11}$ A secondary, albeit non-determinative, benefit of PVE during this current pandemic is the extension of the window to surgery.

When surgery is planned, in keeping with institutional policy, all patients are tested for COVID-19 within 24 hours of surgery. Patients with positive tests will have their hepatectomy postponed. The decision to avoid major surgery in COVID-19 positive patients is based on limited data on outcomes of elective surgery in this patient population, where rates of ICU admission and postoperative mortality were $44.1 \%$ and $20.5 \%$, respectively. ${ }^{12}$ Infected patients need to have fully recovered from their illness (ie, no longer exhibiting signs and/or symptoms of COVID-19) and be deemed safe for surgery based on consensus determination by the surgical and anesthesia teams, with input from pulmonary or infectious disease physicians when necessary, before surgery is entertained. Patients deemed unfit for surgery still may instead be referred for liver-directed therapy as above discussed. When surgery is pursued, the choice of surgical approach remains individualized. We continue to offer minimally invasive hepatectomy, including for anatomic resection, because of the advantage of reduced hospital length of stay and faster return to baseline function for patients. To ensure the safety of the operating room team, institutional OR guidelines are strictly followed. Standardized enhanced recovery after liver surgery care pathways further optimize postoperative outcomes and facilitate early return to intended oncologic therapy. ${ }^{13}$

\section{Conclusions}

In times of crisis like the current COVID-19 pandemic, a coordinated and thoughtful approach to the management 
of malignancies, including that of $\mathrm{HCC}$, is necessary to maintain good oncologic outcomes while preserving critical resources to deal with a potential surge. A strategy that delays surgery to a safer operative window when possible, combined with careful patient selection and meticulous surgical technique, are essential to achieving these dual goals. When the time comes to begin transitioning from COVID-19 readiness to COVID-19 recovery, it will be important to remain vigilant and flexible in our care delivery so as to meet the challenges imposed on our healthcare system and our patients alike.

\section{Disclosure}

The authors report no conflicts of interest in this work.

\section{References}

1. Tzeng CD, Teshome M, Katz MHG, et al. Cancer surgery scheduling during and after the COVID-19 first wave: the MD Anderson cancer center experience. Ann Surg. 2020;272(2):e106-e111. doi:10.1097/ SLA.0000000000004092

2. Tzeng CD, Tran Cao HS, Roland CL, et al. Surgical decision-making and prioritization for cancer patients at the onset of the COIVD-19 pandemic: a multidisciplinary approach. Surg Oncol. 2020;34: 182-185. doi:10.1016/j.suronc.2020.04.029

3. Pryor A. SAGES and EAES recommendations regarding surgical response to COVID-19 crisis. Available from: https://www.sages.org/ recommendations-surgical-response-covid-19/. Accessed May 2, 2020.

4. COVID-19 and solid organ transplant. Available from: https://unos. org/covid/. Accessed May 3, 2020.
5. Ercolani G. Predictive indices of morbidity and mortality after liver resection. Ann Surg. 2006;244(4):635-637. doi:10.1097/01.sla.000 0239644.28302 .16

6. Ripoll C, Groszmann R, Garcia-Tsao G, et al. Hepatic venous pressure gradient predicts clinical decompensation in patients with compensated cirrhosis. Gastroenterology. 2007;133(2):481-488. doi:10. 1053/j.gastro.2007.05.024

7. National Comprehensive Cancer Network. Hepatobiliary cancers (Version 1.2020). Available from: https://www.nccn.org/profes sionals/physician_gls/pdf/hepatobiliary.pdf. Accessed May 3, 2020.

8. Guglielmi A, Ruzzenente A, Conci S, Valdegamberi A, Iacono C. How much remnant is enough in liver resection? Dig Surg. 2012;29 (1):6-17. doi:10.1159/000335713

9. Abdalla EK, Hicks ME, Vauthey JN. Portal vein embolization: rationale, technique and future prospects. Br J Surg. 2001;88(2):165-175. doi:10.1046/j.1365-2168.2001.01658.x

10. Niekamp AS, Huang SY, Mahvash A, et al. Hepatic vein embolization after portal vein embolization to induce additional liver hypertrophy in patients with metastatic colorectal carcinoma. Eur Radiol. 2020:1-7.

11. Teo JY, Allen JC Jr, Ng DC, et al. A systematic review of contralateral liver lobe hypertrophy after unilobar selective internal radiation therapy with Y90. HPB (Oxford). 2016;18(1):7-12. doi:10.1016/j. hpb.2015.07.002

12. Lei S, Jiang F, Su W, et al. Clinical characteristics and outcomes of patients undergoing surgeries during the incubation period of COVID-19 infection. EClinicalMedicine. 2020;21:100331. doi:10.10 16/j.eclinm.2020.100331

13. Day RW, Cleeland CS, Wang XS, et al. Patient-reported outcomes accurately measure the value of an enhanced recovery program in liver surgery. J Am Coll Surg. 2015;221(6):1023-1030. doi:10.1016/j. jamcollsurg.2015.09.011
Journal of Hepatocellular Carcinoma

\section{Publish your work in this journal}

The Journal of Hepatocellular Carcinoma is an international, peerreviewed, open access journal that offers a platform for the dissemination and study of clinical, translational and basic research findings in this rapidly developing field. Development in areas including, but not limited to, epidemiology, vaccination, hepatitis therapy, pathology

\section{Dovepress}

and molecular tumor classification and prognostication are al considered for publication. The manuscript management system is completely online and includes a very quick and fair peer-review system, which is all easy to use. Visit http://www.dovepress.com/ testimonials.php to read real quotes from published authors. 